\title{
Sharing of ARQ Slots in Gilbert-Elliot Channels
}

\author{
Chih-Hsien Hou, Jin-Fu Chang, and De-Yi Chen
}

\begin{abstract}
This letter treats the problem of $m$ transmission slots sharing a pool of $n$ automatic repeat request slots. Two cases are treated. In one, these $m$ slots belong to the same basestation-terminal pair, i.e., a frequency-division multiplexing channel. In the other, they are used by $m$ different basestation-terminal pairs, i.e., a time-division multiplexing channel. The smallest $n$ to satisfy loss and delay requirement is determined.
\end{abstract}

Index Terms-Automatic repeat request (ARQ), fading channels, mobile communications.

\section{INTRODUCTION}

I $\mathrm{N}$ THE LAST decade, mobile wireless communications is, no doubt, one of the fastest emerging technologies. It has rapidly grown from the first-generation pure circuit-switched telephony service to the second generation of having both circuit-mode voice and packet-mode data. In the soon-to-be-deployed third generation, more advanced and sophisticated multimedia-type integrated services are highly anticipated. This is also an enabling technology to make the extension of the Internet to the wireless domain a doable work. But data integrity is an issue that one should not overlook in the extension. In this letter, we focus on the assurance of data integrity in wireless channels. Errors in a wireless channel tend to be bursty and correlative. Among the many works to model bursty and correlative errors in wireless channels, a frequently employed model is the Gilbert-Elliot channel [1], [2] which we also adopt for this letter.

There are many methods to enhance data integrity ranging from forward-error correction (FEC) and automatic repeat request (ARQ), to a hybrid of them. Please consult [3] and [4] for a review of error-control techniques. In this letter, we concentrate on the use of pure ARQ as a means to recover a data cell from loss within its lifetime or freshness. We further focus on error control for data sent from a basestation to mobile terminals. We address the problem where a total of $m$ transmission slots are to share a common pool of $n$ ARQ slots, and try to explore if there should be a minimal number of ARQ slots to fulfill both the requirements of loss and time constraint.

Two scenarios are treated in this letter. In one, these $m$ transmission slots belong solely to one basestation-terminal

Paper approved by K. K. Leung, the Editor for Wireless Network Access and Performance of the IEEE Communications Society. Manuscript received July 16, 2001; revised October 25, 2002 and November 27, 2003. This work was supported in part by the National Science Council, Taiwan under Grant NSC902213-E-260-011(2001).

C.-H. Hou and D.-Y. Chen were with the Graduate Institute of Communication Engineering, National Taiwan University, Taipei, Taiwan 107, R.O.C. They are now with Quanta Computer Inc., Tao Yuan, Taiwan 333, R.O.C.

J.-F. Chang is with the Department of Electrical Engineering, National Chi-Nan University, Puli, Nantou, Taiwan 545, R.O.C. (e-mail: jfchang@ @ncnu.edu.tw).

Digital Object Identifier 10.1109/TCOMM.2004.838680

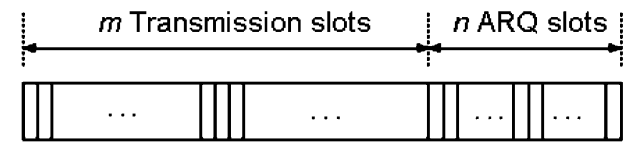

Fig. 1. Frame structure.

pair. This is the case in which downlink transmission slots are arranged in the manner of frequency-division multiplexing (FDM). In the other, they are uniquely assigned to $m$ different basestation-terminal pairs to correspond to the arrangement of time-division multiplexing (TDM). Among the traditional ARQ protocols: stop-and-wait (SW), go-back-N (GBN), and selective repeat (SR); we choose to use the most efficient SR ARQ.

\section{THE FDM SCENARIO}

By convention, the channel time is sliced into slots so that each slot can accommodate the transmission of a data unit, e.g., packet or cell. Depicted in Fig. 1 is the scenario we are considering. That is, we are assuming a frame time of $(m+n)$ slots in an FDM channel.

Regarding time constraint, we assume that each data unit must be successfully transmitted in $k$ frame times; otherwise, it is discarded. Mathematical analysis of this FDM arrangement is by no means trivial. The complication that lies in our FDM problem is manyfold. First, the Gilbert-Elliot channel is correlative in time. Second, among a group of slots that the link owns, we have to know not only whether the transmission in a particular slot has failed, but also exactly how many other slots have failed. Having this information, we then determine how many cells need to be retransmitted via the ARQ protocol. Third, although knowing if a particular slot transmission has succeeded, we need only to know whether that slot is "good" or "bad," to know the status of the rest of the slots is a much more complicated matter, since their slot conditions are correlative. In [5], Yee and Weldon studied the performance of error-correcting codes on a Gilbert channel. We benefited from the results they obtained regarding channel-state variation.

The technique developed in [5] was successfully applied to derive the generating function or $z$-transfrom of cell loss and delay distributions. Please consult [6] for detail derivations.

We have so far assumed an environment of zero round-trip propagation time. That is, acknowledgment returns from the receiving party immediately, with no delay. But, in practice, in a large bandwidth-delay product in particular, such as a satellite environment, the round-trip delay may not be small enough to be negligible. Let the value of round-trip propagation time be equal to $t_{r}$ slots. In this situation, when the channel is up to a new $n$-slot ARQ region, the information is used to determine who is to be granted access to these $n$ ARQ slots that correspond to the transmissions occurring in the $\left(t_{r}+1\right)$ th, $\left(t_{r}+\right.$ 


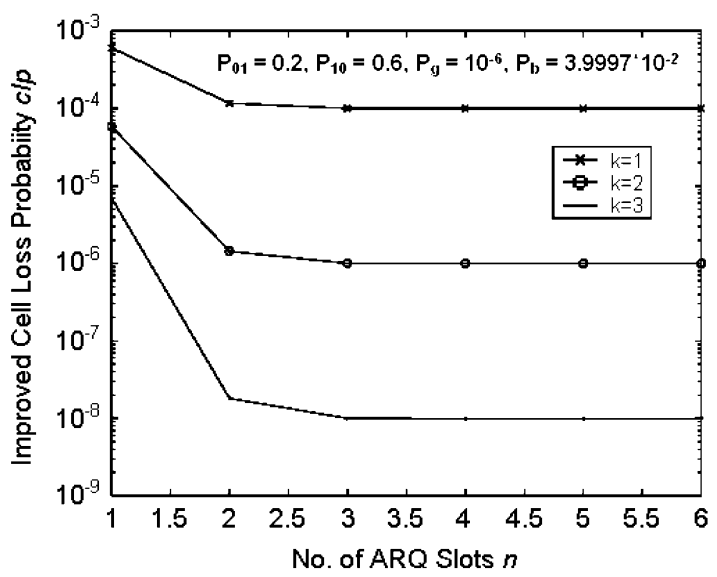

(a)

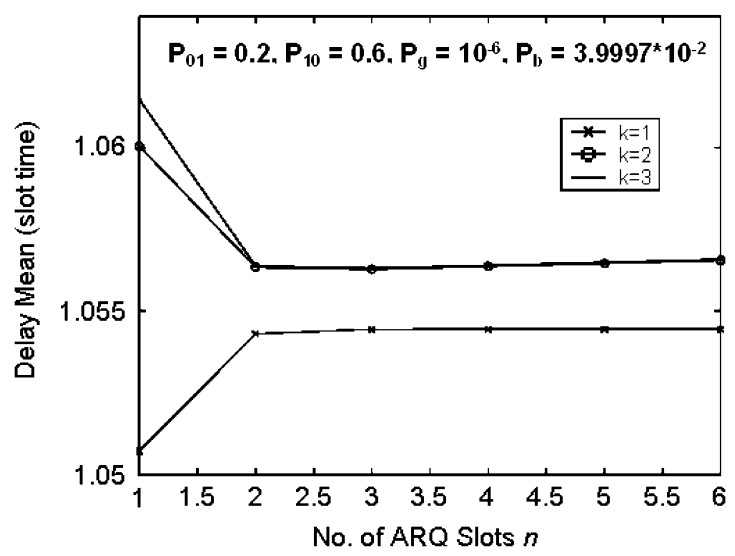

(b)

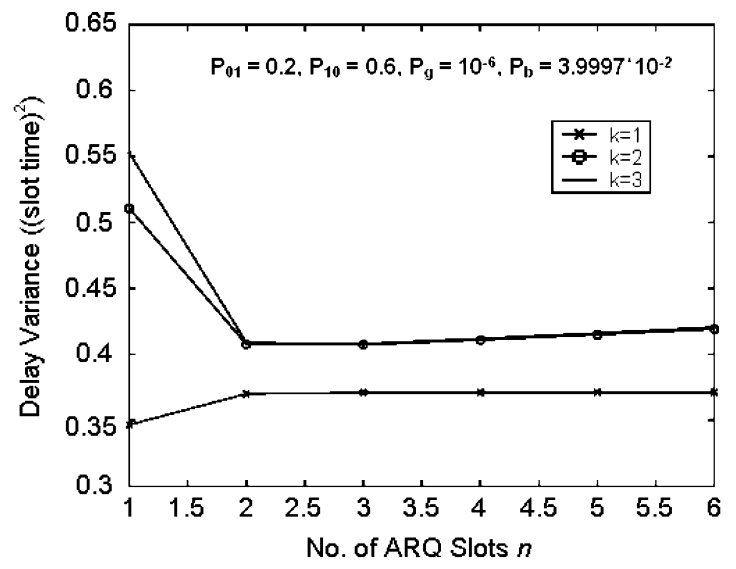

(c)

Fig. 2. Performance of $m=10$ under $k=1$, raw cell error probability $=0.01$ and $t_{r}=0$.

$2)$ th $, \ldots,\left(t_{r}+n\right)$ th $, \ldots,\left(t_{r}+m+n\right)$ th earlier slots. The analysis needs to be modified taking this time lag into account. We shall skip the details and refer them to [7], where only $k=1$ is treated. But if our concern is to see if how many ARQ slots are necessary to reduce the loss probability to a satisfactory level, round-trip delay does not really have an effect. Of course, delay may be largely affected. We shall witness the effect of round-trip delay on loss and delay in the numerical examples that follow.

Fig. 2(a) shows an example of $m=10$ under the raw cell error probability $10^{-2}, k=1,2,3$, and zero round-trip propagation time, i.e., $t_{r}=0$. The Gilbert-Elliot channel parameters leading to this $10^{-2}$ cell error probability are $p_{01}=0.2, p_{10}=$ $0.6, p_{g}=10^{-6}, p_{b}=3.9997 \times 10^{-2}$. In Fig. 2(a), we plot the reduced cell loss probability against the number of ARQ slots employed. We observed that for an $m=10$ setup, a three-slot ARQ region may have reduced the cell error rate by two orders of magnitude; but more ARQ slots do not seem to offer any further reduction. This cell-loss rate floor is due mainly to the $k=1$ time constraint. But the floor can be pushed further down when the time constraint is relaxed from $k=1$ to $k=2$ or 3 using perhaps one more ARQ slot.

The delay mean and variance plotted in Fig. 2(b)-(c) are consistent with that in Fig. 2(a), except for $k=2$ and $k=3$ when $n$ is increased from one to two. Although their actual values really do not differ much, e.g., the delay means are all in the order of 1.0, this microscopic exception is still explainable. The dis- tinction between $k=1$ and $k=2,3$ in Fig. 2(b) and (c) seem to say that for $k>1$, using one ARQ slot creates a delay mean or variance larger than that of using two ARQ slots. This is because when only one ARQ slot is used but a cell can be resent in two or three frames, then each additional retransmission consumes one more frame time. But if the number of ARQ slots is increased, then competition to gain access becomes less fierce, and a cell has a better chance of being successfully resent in the ARQ region of the first frame. It is less likely to need to go to another frame for a successful retransmission.

Now we come to investigate the effect of nonzero round-trip propagation time in Fig. 3, where we plot the same set of performance measures for a $m=20$ configuration by assuming a round-trip delay of $0-50$ slots, i.e., $t_{r}=0-50$. As we have expected, the cell-loss behavior is really indifferent to the introduction of round-trip propagation time, but delay mean and variance do show an increasing trend when the round-trip time becomes longer.

\section{THE TDM SCENARIO}

In this TDM arrangement, the $m$-slot region in Fig. 1 is assigned one by one to $m$ basestation-terminal pairs, and the $n$-slot retransmitted or ARQ region is shared by those basestation-terminal pairs awaiting an opportunity for retransmission. We assume that each time an ARQ region has emerged for a 


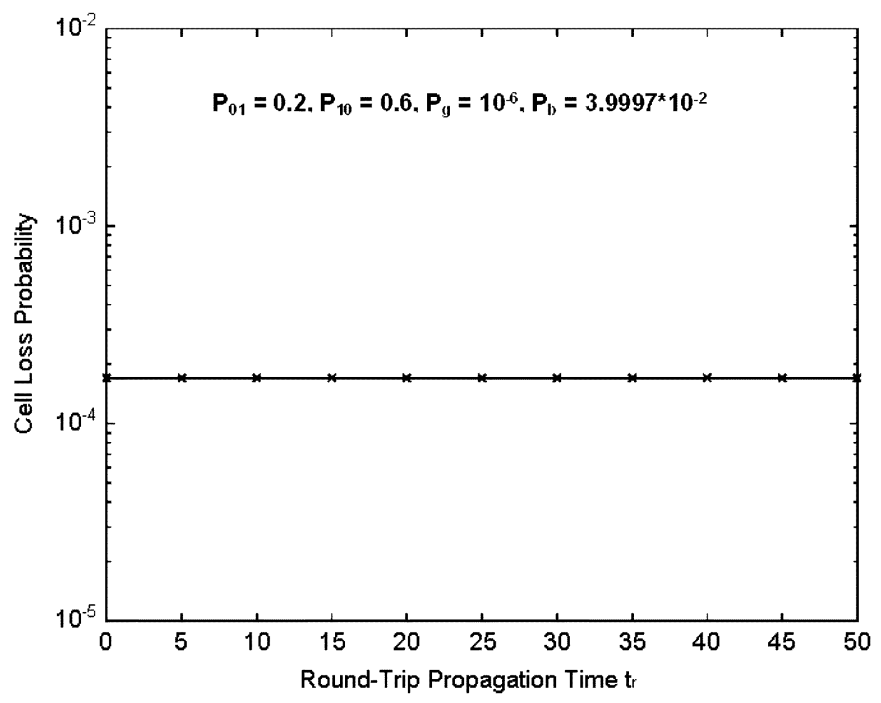

(a)

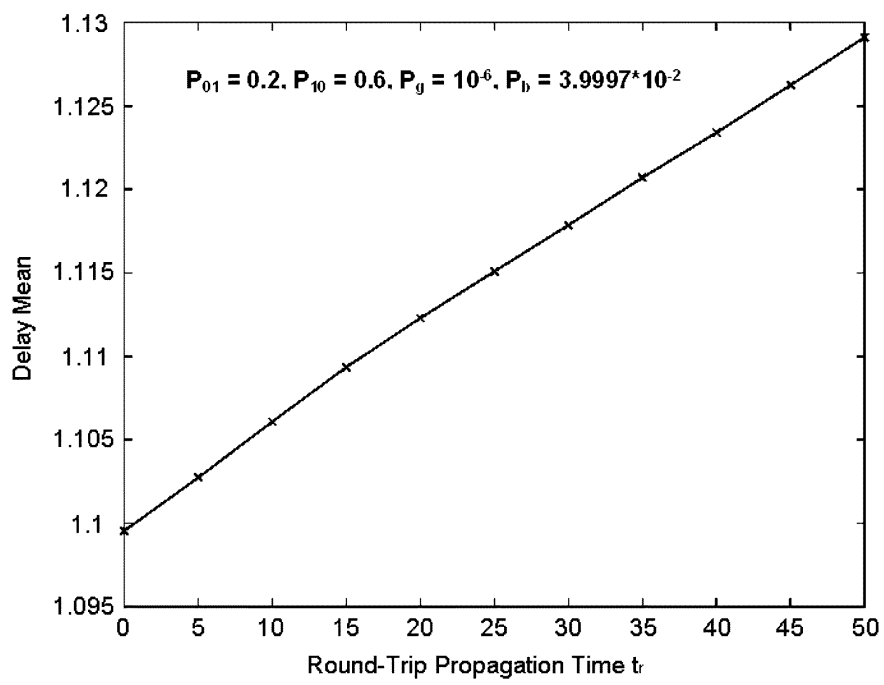

(b)

Fig. 3. Effect of round-trip propagation time.

new round, each basestation-terminal pair (having at least one cell awaiting retransmission) is given the opportunity of no more than one slot. These basestation-terminal pairs are drawn randomly to access the ARQ region. Although channel conditions in different slots appear to be somewhat independent due to their independent movements, the TDM situation becomes more complicated since we are now dealing with a sort of interleaving between $m$ Gilbert-Elliot channels.

In [6], we have conducted approximate mathematical analysis for the case in which each of these $m$ Gilbert-Elliot channels has the same parameter set of $p_{00}, p_{01}, p_{10}$, and $p_{11}$.

In Fig. 4, we again plot cell-loss probability versus $n$ for $m=10$. Simulated results are also provided to verify the quality of approximation. Each simulation is run for a total of 5000 slots. The simulation results are plotted with a $95 \%$ confidence interval. We clearly observe the closeness between approximation and simulation. The downhill behavior and the order of magnitude improvement of Fig. 4 resemble Section II.

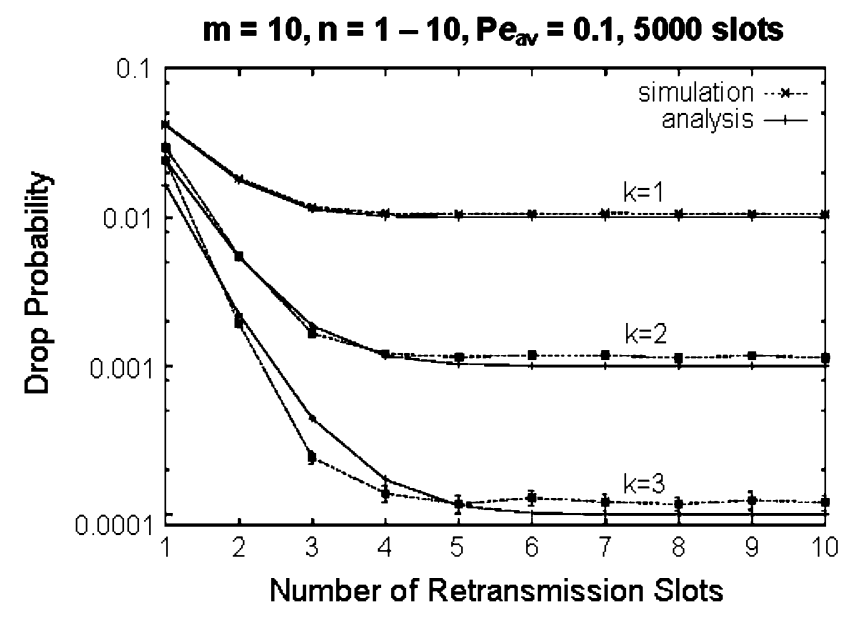

Fig. 4. Cell-loss performance of a ten-connection setup.

\section{CONCLUSION}

We have in this letter investigated the impact of using ARQ error-control techniques on cell-loss performance, amid the influence of correlative Gilbert-Elliot channel errors. Thanks to the groundwork paved by Yee and Weldon [5], we have successfully, although still sophisticatedly, derived the formulas of cell-loss probability and delay mean and variance for the FDM scenario. Due to its further complication, we are able only to obtain a cell-loss result for the TDM scenario through approximation whose accuracy has been checked by simulation and proves to be good.

We may draw the following conclusions from the numerical examples we have presented. First, it is indeed effective for a group of data slots to share only a very few ARQ slots to bring cell-loss probability to one to two orders of magnitude less. Second, under a fixed time constraint of $k$ frames, a cell-loss probability floor prevails regardless the number of ARQ slots that one wishes to invest. To push this floor further down is possible only if more delay can be tolerated. Third, the reduction in cell loss, of course, is achieved at the cost of longer delay and larger variance. Fourth, although round-trip propagation time imposes an impact on delay behavior, it does nothing to help or hurt cell-loss rate.

\section{REFERENCES}

[1] E. N. Gilbert, "Capacity of a burst-noise channel," Bell Syst. Tech. J., vol. 39, pp. 1253-1265, Sept. 1960.

[2] E. O. Elliot, "Estimates of error rates for codes on burst-noise channels," Bell Syst. Tech. J., vol. 42, pp. 1977-1997, Sept. 1963.

[3] H. Liu, H. Ma, M. El Zarki, and S. Gupta, "Error control schemes for networks: An overview," Mobile Networks, Applicat., vol. 2, pp. 167-182, 1997.

[4] S. Lin and D. J. Costello, Jr., Error Control Coding: Fundamentals and Applications. Englewood Cliffs, NJ: Prentice-Hall, 1983.

[5] J. R. Yee and E. J. Weldon, Jr., "Evaluation of the performance of errorcorrecting codes on a Gilbert channel," IEEE Trans. Commun., vol. 43, pp. 2316-2323, Aug. 1995.

[6] C.-H. Hou, J.-F. Chang, and D.-Y. Chen. (2002) Sharing of ARQ Slots in Gilbert-Elliot Channel. [Online]. Available: http://www.president.ncnu.edu.tw/chang.pdf

[7] C.-H. Hou, "Performance of selective repeat ARQ protocols on burst error channel for multiple connections," Master's thesis, Grad. Inst Commun. Eng., National Taiwan Univ., Taipei, Taiwan, R.O.C., June 2000 . 